\title{
Pleomorphic Carcinoma of the Lung: Clinicopathological Characteristics and Treatment Outcomes of 20 Cases
}

Gulbanu Horzum Ekinci ${ }^{1}$, Osman Haciomeroglu ${ }^{1}$, Ayse Ersev ${ }^{2}$, Cagatay Tezel $^{3}$, Arzu Cennet Isik $^{4}$, Levent Alpay $^{3}$, Adnan Yilmaz ${ }^{1}$

\begin{abstract}
Background and Aims: Pleomorphic carcinoma is a rare tumor of the lung. The aim of the present study was to evaluate clinicopathological characteristics and treatment outcomes of patients with pleomorphic pulmonary carcinoma.
\end{abstract}

Methods: The records of pathology department from January 2005 to December 2013 were evaluated. Twenty cases of pleomorphic pulmonary carcinoma were identified and studied.

Results: There were 18 male and two female patients. Their age ranged from 38 to 81 years, with the mean age of 63.4 years. Eighteen $(90 \%)$ patients had smoking history. All patients were symptomatic. The most common radiological finding was solitary mass, followed by solitary pulmonary nodule. Fiberoptic bronchoscopy revealed an endobronchial lesion in six $(30 \%)$ patients. Pathological diagnosis of pleomorphic carcinoma was made by bronchoscopic biopsy in two $(10 \%)$ patients and surgical biopsy in $18(90 \%)$ patients. Four (20\%) patients had metastatic disease at the time of diagnosis. Fifteen patients underwent surgical resection. Lobectomy was the most common surgical procedure. Pathologically, the epithelial component of the tumor was squamous cell carcinoma in $13(65 \%)$ patients and adenocarcinoma in $7(35 \%)$ patients. Survival time for 11 patients was six months or shorter than six months. Survival time ranged from 1 month to 97 months, with a median survival time of 6 months.

Conclusions: Pleomorphic carcinoma is a rare tumor of the lung. Pathologic diagnosis of this tumor is usually established by surgical biopsy. The prognosis of patients with pleomorphic pulmonary carcinoma is poor despite complete surgical resection.

Keywords: Lung, pleomorphic carcinoma, prognosis, treatment

From: ${ }^{1}$ Department of Pulmonology, ${ }^{2}$ Department of Pathology, ${ }^{3}$ Department of Thoracic Surgery, ${ }^{4}$ Department of Internal Medicine Süreyyapaşa Chest Diseases and Thoracic Surgery Training and Research Hospital,

Correspondence: Dr GH Ekinci, Süreyyapaşa Gögüs Hastalıkları ve Göğüs, Cerrahisi Eğitim ve Araştırma Hastanesi Maltepe, Istanbul, Turkey. Fax No: +90 21642141 30, E-mail: gulbanuh@ hotmail.com 


\section{INTRODUCTION}

Pleomorphic carcinoma (PC) is a rare tumor of the lung. It accounts for $0.1-1 \%$ of all pulmonary malignant tumors $(1,2)$. According to the 2004 World Health Organization classification of lung tumors, pleomorphic carcinoma is one of five subgroups of pulmonary sarcomatoid carcinoma (3). An accurate preoperative diagnosis of this tumor by bronchoscopic or transthoracic needle biopsy is difficult due to the heterogeneity of the tumor (4). Many case reports but very few studies have been published in the literature regarding clinical and pathological characteristics of pulmonary pleomorphic carcinoma. As a result its clinical and pathological characteristics are not well known $(2,5)$. The purpose of the present study was to evaluate clinicopathological characteristics and treatment outcomes of patients with pleomorphic pulmonary carcinoma.

\section{METHODS}

This retrospective study was conducted at Süreyyapaşa Chest Diseases and Thoracic Surgery Training and Research Hospital, Istanbul, Turkey. The study was approved by the local scientific committee of institute. The records of pathology department from January 2005 to December 2013 were reviewed. Twenty-one cases of pleomorphic pulmonary carcinoma were identified during this period. All the pathological material was reexamined by an expertise pathologist. Hematoxylin and eosin stained slides were present in all cases. Immunohistochemical staining was performed in 18 cases. The diagnosis of pulmonary pleomorphic carcinoma was based on the criteria set by World Health Organization (3). One patient was excluded from this study because the tumor didn't meet the criteria set by World Health Organization. Thus, the present study included 20 cases of pleomorphic carcinoma. 
The patients' clinical files were evaluated for the following: age, gender, history of smoking, symptoms, radiological features, diagnostic investigations, pathological findings, stage of the tumor, treatment, and outcomes.

All patients underwent routine laboratory studies, electrocardiography, chest-x ray, computed tomography (CT) of the thorax, and fiberoptic bronchoscopy. CT-guided transthoracic fine needle aspiration (TFNA) was performed in nine patients. Pulmonary function tests were performed in patients who underwent a surgical procedure. Metastatic disease was investigated by positron emission tomography (PET-CT) in 18 patients, by magnetic resonance of the brain in 10 patients, by $\mathrm{CT}$ of the brain in one patient, CT of the abdomen in two patients, and bone scintigraphy in two patients. Fifteen patients underwent surgical resection. The standard surgical technique was used and accompanied by routine systemic dissection or sampling of the hilar and mediastinal lymph nodes in these patients. A surgical biopsy was performed in three patients. The clinical and pathologic staging of the tumor were made according to the seventh edition of international TNM staging system. Follow-up information was completed on December 2014. The duration of overall survival was defined as the interval between the day of the operation or diagnosis and the date of death or the last follow-up date.

Statistical analysis: The overall survival rate was calculated by using the Kaplan-Meier method.

\section{RESULTS}

There were $18(90 \%)$ male and $2(10 \%)$ female patients (male to female ratio, 9 to1). The mean age was 63.4 years (range 38-81 years). Eighteen (90\%) patients had smoking history. 
All patients were symptomatic. The most common symptom was chest pain, followed by dyspnea. The clinical data was given in table 1 .

Chest X-ray showed a solitary mass in 13 patients, a solitary pulmonary nodule in four, atelectasis in one, cavitation in one, and cavitation and infiltration in one. CT scan of the thorax demonstrated a solitary mass in 14 patients, a solitary pulmonary nodule in three, multiple pulmonary nodules in one, atelectasis and mass in one, and cavitation and infiltration in one. Tumor size on CT scan ranged from $10 \mathrm{~mm}$ to $96 \mathrm{~mm}$, with the mean size of 51.7 $\mathrm{mm}$. While the size was smaller than $5 \mathrm{~cm}$ in five $(25 \%)$ cases, it was $5 \mathrm{~cm}$ or larger than 5 $\mathrm{cm}$ in $15(75 \%)$ cases. All patients underwent flexible bronchoscopy. It revealed endobronchial lesion in six $(30 \%)$ patients and normal endobronchial appearance in 14 (70 \%) patients. According to $\mathrm{CT}$ and/or bronchoscopy findings, the tumors were located in the right upper lobe $(n=6)$, left upper lobe $(n=5)$, left lower lobe $(n=3)$, right lower lobe $(n=2)$, right upper and lower lobes $(n=1)$, left lower and right lower lobes $(n=1)$, right middle lobe $(\mathrm{n}=1)$, and right bronchus intermedius $(\mathrm{n}=1)$. PET-CT was performed in 18 patients. SUVmax value on PET-CT was ranged from 5.7 to 36, with the mean SUVmax value of 14.1.

Pleomorphic carcinoma was diagnosed in only two (10\%) patients by preoperative diagnostic procedures. Pathological diagnosis was established by surgical biopsy in three patients and by surgical resection in 15 patients. Pathologically, the epithelial component of the tumor was squamous cell carcinoma in $13(65 \%)$ patients and adenocarcinoma in 7 (35 \%) patients. Pathological results of flexible bronchoscopy and CT-guided TFNA were given in table 2 .

The data of stage, treatment, follow-up, and survival on 20 patients of pleomorphic carcinoma were summarized in table 3 . Four $(20 \%)$ patients had metastatic disease at the time of diagnosis. Metastatic sites included lung $(n=1)$, bone $(n=1)$, adrenal gland $(n=1)$, and brain $(n=1)$. While three patients were treat with chemotherapy, the other refused treatment. 
One patient was medically inoperable because of poor cardiopulmonary status. He was treated with chemotherapy. Remaining 15 patients underwent surgical resection. Operations included 11 lobectomies, two bilobectomies inferior, and two pneumonectomies. Surgical resection was accompanied by routine systemic dissection in 14 patients and sampling of the hilar lymph nodes in one patient. No mortality occurred in the postoperative period. Four patients had prolonged air leak and the patients were treated with chest tube placement. While six patients received adjuvant chemotherapy, two patients received adjuvant chemoradiotherapy. Survival time ranged from 1 month to 97 months, with the median survival time of 6 months. This time for 11 patients was six months or shorter than six months. Three patients were alive at the time of evaluation. Figure 1 presented the cumulative survival graph.

\section{DISCUSSION}

According to the 2004 World Health Organization classification of lung tumors, pleomorphic carcinoma is one of five subgroups of sarcomatoid carcinoma (3). Histologically, pleomorphic carcinoma is defined as either a non-small cell lung carcinoma combined with neoplastic spindle and/or giant cells or a carcinoma that consists of only spindle and giant cells. At least $10 \%$ of the carcinoma should comprise spindle and/or giant cells for it to be classified as a pleomorphic carcinoma $(3,6)$. It accounts for $0.1-1 \%$ of all malignant lung tumors $(1,2)$. Nearly 1100 cases with primary pulmonary malignancy have been diagnosed annually in our center. According to these results, its incidence is $0.2 \%$ in our center.

Pleomorphic carcinoma predominantly occurs in elderly men who smoke heavy, as similar as our findings (7-9). The male:female ratio ranges from 2.3:1 to $10.3: 1(5,7)$. The mean age at diagnosis is $57-66$ years $(9,10)$. There is strong association of this tumor with cigarette smoking. The rate of the smoker patients is between $80 \%-95 \%(4,5)$. Most patients 
present with nonspecific pulmonary symptoms including chest pain, cough, hemoptysis, and dyspnea, whereas they can be asymptomatic for a long time $(4-6,11,12)$. Chang et al (13) reported that all patients presented with one or more symptoms. Two previous reports noted that symptoms were seen in $72.7 \%$ and $90 \%$ of the patients $(5,6)$. In our series, all patients were symptomatic. Symptoms are associated with the location and size of the tumor. Pulmonary pleomorphic carcinomas are classified either as central endobronchial type or peripheral parenchymal type on the basis of the location of the tumor. Central type produces several symptoms as a result of bronchial irritation, obstruction or obstructive pneumonia. Peripheral type produces symptoms due to the early pleural and chest wall invasion $(7,11$, 13). Pleomorphic carcinomas tend to be large peripheral tumors, as in our series. They are often found as a large mass, more than $4-5 \mathrm{~cm}$ in diameter. Large tumors are usually symptomatic $(6,11)$. We conclude that pulmonary symptoms may associated with smoking in many smoker patients.

The radiological findings of pulmonary pleomorphic carcinoma are nonspecific and similar to those of other primary lung cancers. Most patients present with a solitary pulmonary mass or nodule on radiographs and CT. The images frequently demonstrate intratumoral cavities $(6,10,13)$. While the lesions are generally unilateral and solitary, bilateral or multiple lesions can be identified on radiographic images (11). There is a predilection for peripheral location and upper lobes $(7,10)$. Those findings are consistent with those in our series.

The preoperative diagnosis of pleomorphic carcinoma is very difficult because the diagnostic value of preoperative diagnostic methods including bronchoscopy and transthoracic needle aspiration is limited $(4-7,14)$. Yamamato et al (4) reported that none of the patients had a preoperative diagnosis of pleomorphic carcinoma. Ito et al (6) noted that transbronchial biopsy was diagnostic in three of 22 cases and there were no cases diagnosed 
with cytological examination. This is due to the heterogeneity and rare incidence of this tumor (7, 14). Bronchoscopy and transthoracic fine needle aspiration can yield small amount of tissue for pathological examination. Also, they often demonstrate only one component of the tumor. As a result, a definitive diagnosis is established based on examination of surgical specimens in the majority of the cases $(5,7)$. In our series, only two patients had preoperative diagnosis of pleomorphic carcinoma. The definitive diagnosis was established with examination of surgical specimens in remaining 18 patients.

Pleomorphic carcinoma presents an aggressive behavior. At the time of presentation, local invasion of adjacent structures, metastasis to hilar and mediastinal lymph nodes, and distant metastases are frequent $(5-7,13)$. Chang et al (13) reported that among seven of the patients who underwent surgical resection, four of them had mediastinum, pleura and chest wall invasions and three of them had regional lymph node metastases. Mochizuki et al (9) noted that vascular invasion and lymph node metastases were observed in $86 \%$ and $41 \%$ of the patients, respectively. They reported that $7 \%$ of the patients had the pathological stage IV. According to Fishback's report (11), $12 \%$ of the patients had stage IV lesions at the time of diagnosis. In our series, four patients had chest wall invasion. There were N1 lymph node metastases in two patients and N2 lymph node metastases in two patients. Four patients had distant metastases at the time of presentation. Pleural effusion was diagnosed in one patient with distant metastases.

Complete surgical resection of the tumor with clear tumor margins is the treatment of choice in especially early stage patients with pulmonary pleomorphic carcinoma $(4-7,9,11$, 13). Raveglia et al (5) suggested that surgery for preoperatively proven pulmonary pleomorphic carcinoma should be restricted to N0 patients. Adjuvant and neoadjuvant chemotherapy and radiotherapy are controversial and they can be considered in selected cases $(4-7,13)$. The rate of local recurrence and distant metastases after surgery are high $(6,7,9$, 
15). Mochizuki et al (9) reported that twenty-four patients had died as a result of distant metastases or recurrence 27 days to 36 months after surgery. Raveglia et al (5) noted that sixteen of 20 patients died from early distant metastases after surgery. Pleomorphic carcinoma have a worse prognosis than the other non-small cell carcinoma cases $(7,9)$. The overall 5year survival rate, median survival time, disease-free survival rate, and median disease-free survival time were $36.7 \%, 22.8$ months, $40.8 \%$, and 14.7 months, respectively (9). Five-year overall survival was found as $39.2 \%$ in other study (7). Prognosis can be associated with several factors. These factors include location, size, and stage of the tumor, the presence of distant metastases and lymph node metastases $(5,9,11,13)$. The central type have better prognosis than peripheral type because of their limited extension to the surrounding parenchyma and there are early symptoms. Early dissemination is more frequent in the peripheral type (13). The size of tumor greater than $5 \mathrm{~cm}$, stage greater than 1 at presentation, the presence of any lymph node metastases and distant metastases indicate a poor prognosis for survival $(5,11)$. In our series, most patients had one or more of poor prognostic factors. The median survival time was 6 months. Survival time for 11 patients was six months or shorter than six months.

In conclusion, pleomorphic carcinoma is a rare tumor of the lung. Pathologic diagnosis of this tumor is usually established by surgical biopsy. The prognosis of patients with pleomorphic pulmonary carcinoma is poor despite complete surgical resection. 


\section{REFERENCES}

1. Kikuchi R, Isowa N. Tokuyasu H, Kawasaki Y, Onuma H, Miura H. Three cases of resected pleomorphic carcinoma. Ann Thorac Cardiovasc Surg 2010; 16: 264-9.

2. Fujioka S, Nakamura H, Adachi Y, Miwa K, Haruki T, Taniguchi Y et al. Pleomorphic carcinoma of the lung in which the sarcomatous element grew rapidly: A case report. Ann Thorac Cardiovasc Surg 2009; 15: 111-4.

3. Travis WD, Brambilla E, Müller-Hermelink HK et al. World Health Organization classification of tumors. Pathology and genetics of tumors of the lung, pleura, thymus and heart. 2004. $4^{\text {th }}$ edition. IARC Press, Lyon.

4. Yamamato S, Hamatake D, Ueno T, Higuchi T, Hşiratsuka M, Shiraishi T et al. Clinicopathological investigation of pulmonary pleomorphic carcinoma. Eur $\mathrm{J}$ Cardiothorac Surg 2007; 32: 873-6.

5. Raveglia F, Mezzetti M, Panigalli T, Furia S, Giuliani L, Conforti S et al. Personal experience in surgical management of pulmonary pleomorphic carcinoma. Ann Thorac Surg 2004; 78: 1742-7.

6. Ito K, Oizumi S, Fukumoto S, Harada M, Ishida T, Fujita Y et al. Clinical characteristics of pleomorphic carcinoma of the lung. Lung Cancer 2010; 68: 204-10.

7. Yuki T, Sakuma T, Ohbayashi C, Yoshimura M, Tsubota N, Okita Y et al. Pleomorphic carcinoma of the lung: A surgical outcome. J Thorac Cardiovasc Surg 2007; 134: 399-404.

8. Tsubata Y, Sutani A, Okimoto T, Matsuura M, Murakami I, Usuda R et al. Tumor angiogenesis in 75 cases of pleomorphic carcinoma of the lung. Anticancer Res 2012; 32: $3331-8$. 
9. Mochizuki T, Ishii G, Nagai K, Yoshida J, Nishimura M, Mizuno T et al. Pleomorphic carcinoma of the lung. Clinicopathological characteristics of 70 cases. Am J Surg Pathol 2008; 32: 1727-35.

10. Kim TS, Han J, Lee KS, Jeong YJ, Kwak SH, Byun HS et al. CT findings of surgically resected pleomorphic carcinoma of the lung in 30 patients. AJR 2005; 185: 120-5.

11. Fishback NF, Travis WD, Moran CA, Guinee DG, McCarthy WF, Koss MN. Pleomorphic (spindle/giant cell) carcinoma of the lung. Cancer 1994; 73: 2035-45.

12 Chen F, Sonobe M, Sato T, Sakai H, Huang CL, Bando T et al. Clinicopathological characteristics of surgically resected pulmonary pleomorphic carcinoma. Eur J Cardiothorac Surg 2012; 41: 1037-42.

13. Chang YL, Lee YC, Shih JY, Wu CT. Pulmonary pleomorphic (spindle9 cell carcinoma: peculiar clinicopathological manifestations different from ordinary nonsmall cell carcinoma. Lung Cancer 2001; 34: 91-7.

14. Choi HS, Seol .h, Heo II Y, Jung CW, Cho SY, Park S et al. Fine-needle aspiration cytology of pleomorphic carcinomas of the lung. Korean J Pathol 2012; 46: 576-82.

15. Gonçalves MJ, Mendes MM, Joao F, Lpoes JM, Honavar M. Primary pleomorphic sarcoma of lung-11 year survival. Pneumologia 2011; 17: 44-7. 
Table 1: The clinical data of the patients

\begin{tabular}{lll}
\hline & $\mathbf{N}$ & $\mathbf{\%}$ \\
\hline Male & 18 & 90 \\
Female & 2 & 10 \\
Mean age (range) years & $63.4(38-81)$ & \\
Age Groups & & \\
$<40$ years & 1 & 5 \\
$40-60$ years & 8 & 40 \\
$\geq 60$ years & 11 & 55 \\
Smoking History & & \\
Yes & 18 & 90 \\
No & 2 & 10 \\
Mean Smoking Duration (range), years & $59.5(25-150)$ & \\
Symptoms & & \\
Chest pain & 9 & 45 \\
Dyspnea & 8 & 40 \\
Cough & 7 & 35 \\
Hemoptysis & 5 & 25 \\
Sputum production & 3 & 15 \\
Constitutional & 4 & 20 \\
\hline
\end{tabular}

Table 2. The results of flexible bronchoscopy and CT-guided TFNA

\begin{tabular}{lll}
\hline Bronchoscopy $(\mathbf{n = 2 0})$ & $\mathbf{n}$ & $\mathbf{\%}$ \\
\hline Negative & 15 & 75 \\
Pleomorphic carcinoma & 2 & 10 \\
Squamous cell carcinoma & 2 & 10 \\
Non-small cell lung carcinoma & 1 & 5 \\
CT-guided TFNA (n=9) & & \\
Negative & 4 & 44.5 \\
Squamous cell carcinoma & 2 & 22.2 \\
Non-small cell lung carcinoma & 2 & 22.2 \\
Adenocarcinoma & 1 & 11.1 \\
\hline
\end{tabular}


Table 3. The data of stage, treatment follow-up, and survival on 20 patients of pleomorphic carcinoma

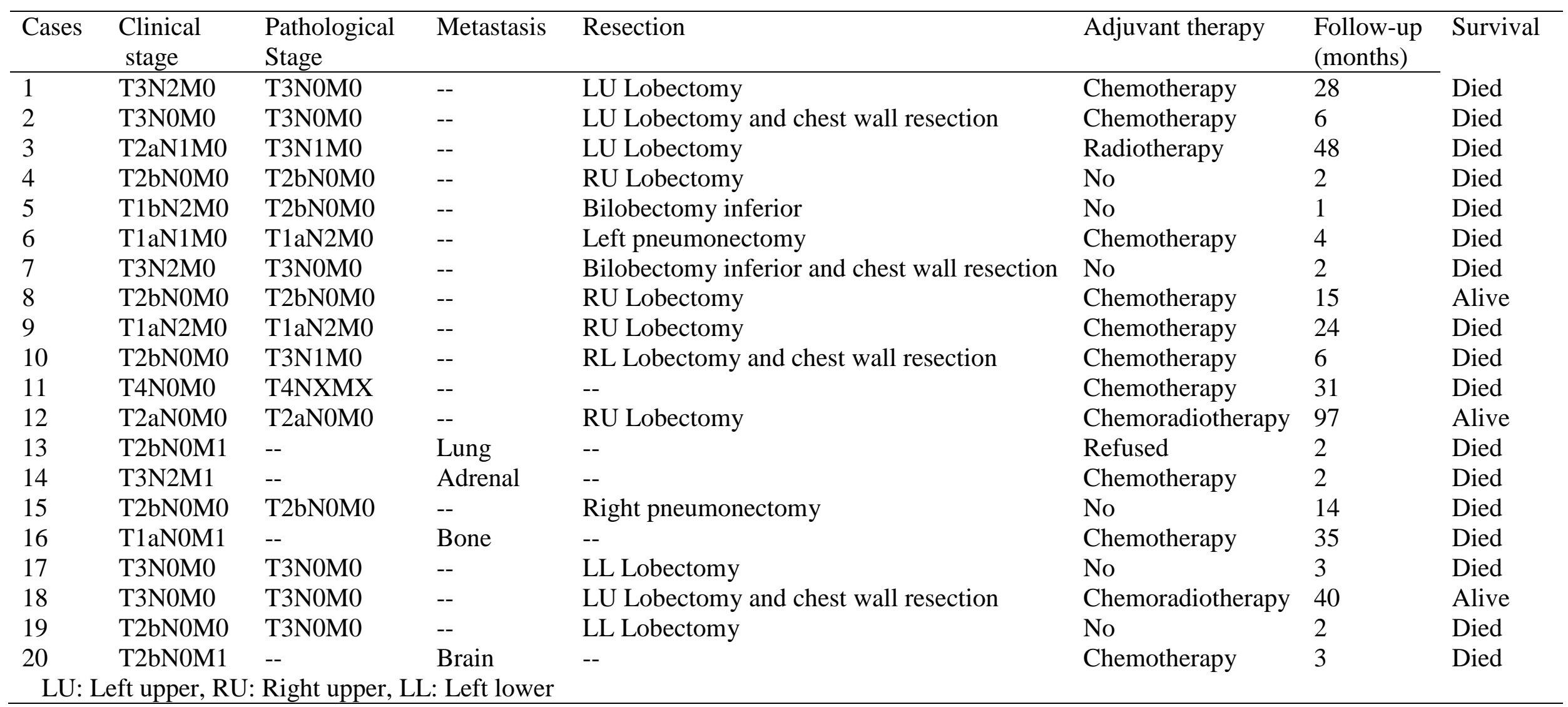




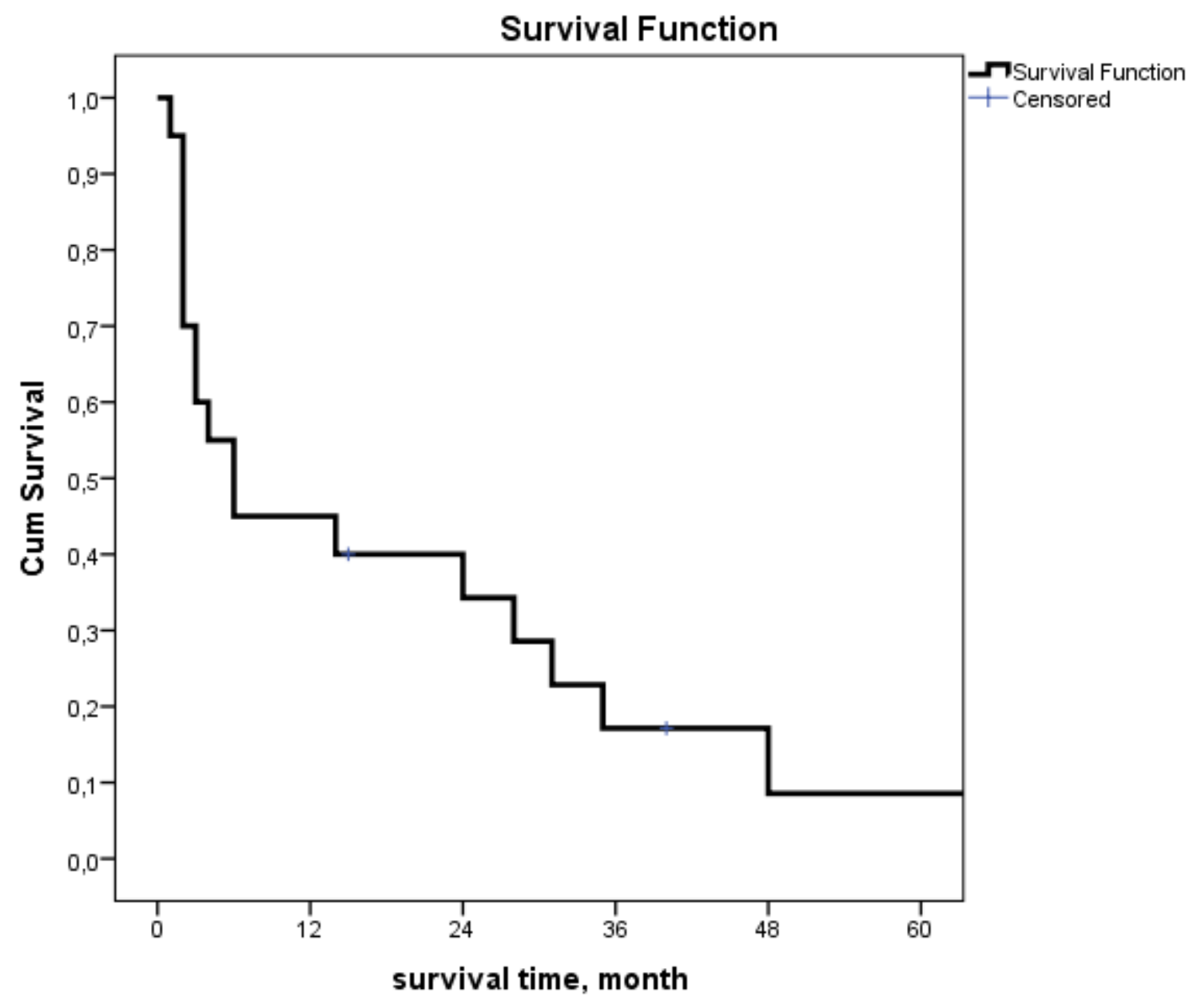

Figure 1. The cumulative survival graph

Figure: The cumulative survival graph. 